\title{
Neoplasia sólida pseudopapilar del páncreas. Reporte de un caso
}

\author{
Ignacio Maldonado Schoijet, ${ }^{1}$ Javiera Vásquez Poblete, ${ }^{2}$ Sergio Álvarez Diaz, ${ }^{3}$ Javier González \\ Moscoso $^{3}$ \\ ${ }^{1}$ Radiólogo Unidad de Cuerpo, Departamento Imagenología, Clinica Dávila; Profesor Adjunto Radiología Universidad Mayor y Universidad de los Andes. \\ ${ }^{2}$ Residente $3^{\circ}$ año Radiología, Universidad Mayor, Clinica Dávila. \\ ${ }^{3}$ Cirujano Adulto, Equipo Cirugía Digestiva y Unidad de Trasplante, Clinica Dávila. \\ Santiago de Chile, Chile.
}

Acta Gastroenterol Latinoam 2020;50(3):345-349

Recibido: 01/12/2019 / Aceptado: 26/06/2020 / Publicado online: 28/09/2020

\section{Resumen}

Introducción. La neoplasia sólida-pseudopapilar del páncreas, es un tumor de origen epitelial del páncreas exocrino, muy infrecuente, que representa el 1-2\% de todos los tumores del páncreas y que afecta principalmente a mujeres jóvenes. Objetivo. Describir los principales hallazgos clinico-imagenológicos de esta entidad poco frecuente en una mujer joven sintomática. Caso clínico. Paciente de sexo femenino de 41 años, sin antecedentes mórbidos, consulta por epigastralgia intermitente de un mes de evolución. La ecotomografía abdominal y resonancia magnética de abdomen, demostraron la presencia de una masa sólido-quística de aspecto neoplásico en el cuerpo-cola del páncreas. El caso fue presentado en comité institucional hepato-biliar, que decidió tratamiento quirúrgico. Discusión. Aunque las neoplasias sólidas-pseudopapilares del páncreas son tumores pancreáticos de baja prevalencia, es importante que el radiólogo y los clínicos estén familiarizados con esta entidad, que debe ser considerada en el diagnóstico diferencial en aquellas lesiones sólido-quisticas, especialmente, en mujeres jóvenes, dado que

Correspondencia: Ignacio Maldonado Schoijet

Av Recoleta \# 464 (Zip: 8431657), Departamento de Imagenología,

Clínica Dávila. Santiago, Chile

Tel.: +56999641172

Correo electrónico: imschoijet@gmail.com presentan un bajo potencial maligno y muy buen pronóstico con el tratamiento quirúrgico.

Palabras claves. Neoplasia sólida pseudopapilar, tumores pancreáticos, masa sólido-quistica, pancreatectomia parcial.

\section{Solid and papillary epithelial neoplasm of the pancreas. A case report}

\section{Summary}

Background. Solid pseudopapillary neoplasm of the pancreas is an uncommon exocrine pancreatic tumor of epithelial origin, which represents only $1 \%$ to $2 \%$ of all pancreatic tumors and mainly affects young women. Objective. To describe clinical and imaging features of these rare entity in a symptomatic young woman. Case report. 41-year-old female patient, without morbid history, consulted for intermittent epigastralgia of one-month evolution. Abdominal ultrasound and magnetic resonance imaging demonstrated the presence of a solid-cystic mass of neoplastic appearance in the body-tail of the pancreas. The case was presented to the institutional hepato-biliary committee where surgical resection was decided. Discussion. Although solid pseudopapillary neoplasm of the pancreas are very uncommon pancreatic tumors, it is necessary to keep them within the differential diagnosis on the one hand due to their low but existing malignant potential and on the other hand due to their good prognosis with surgical treatment.

Key words. Solid and papillary epithelial neoplasm of the pancreas, pancreatic tumors, cystic mass, partial pancreatectomy, SPEN. 


\section{Abreviaturas}

NSSP: Neoplasia sólida pseudopapilar.

RM: Resoncia magnética.

TCMC: Tomografia computada multicorte.

\section{Introducción}

La neoplasia sólida-pseudopapilar del páncreas (NSPP) es un tumor epitelial del páncreas exocrino que representa sólo el 1-2\% de todos los tumores del páncreas y que ocurre predominantemente en mujeres jóvenes en la segunda y tercera décadas de la vida. ${ }^{1-3}$ Se han publicado algunos casos en hombres, los que tienden a presentarse en un grupo etario mayor y a ser más agresivos que en la población femenina. ${ }^{2}$

La mayoría de las NSSP son tumores benignos con bajo grado de potencial maligno, que suelen presentarse como una voluminosa masa única y heterogénea, que si bien puede localizarse en cualquier segmento pancreático, existe predilección por la cola. ${ }^{2}$ Tienen un muy buen pronóstico con el tratamiento quirúrgico, con una tasa de curación de más del $90 \%$ a 5 años. ${ }^{2,3}$

La mayor parte de los pacientes presentan síntomas inespecíficos al momento del diagnóstico. ${ }^{3}$ El síntoma más frecuente es el dolor abdominal. ${ }^{2,3}$ Han sido descritos las náuseas, vómitos, pérdida de peso, ictericia y sangrado gastrointestinal, pero son muy infrecuentes (menos del $12 \%$ de los casos). ${ }^{2,3}$ Los exámenes de laboratorio suelen ser normales.

\section{Caso clínico}

Paciente de sexo femenino de 41 años, sin antecedentes mórbidos de relevancia, que consulta de forma ambulatoria por epigastralgia intermitente de un mes de evolución. Al examen físico y de laboratorio no hay hallazgos significativos. Se solicitó una ecotomografía de abdomen, que informó la presencia de una masa heterogénea en el cuerpo-cola del páncreas (Figura 1).

Se realizó una resonancia magnética (RM) de abdomen, que confirmó la presencia de una masa sólido-quística hipovascular, encapsulada, dependiente del aspecto anterior del cuerpo-cola del páncreas (Figuras 1 y 2),

Figura 1. RM abdomen. A) T2 Haste coronal. B) Ecotomografia de abdomen en región de epigastrio centrado en el páncreas. C) T1 Fatsat sin contraste axial. D) T1 Fatsat con contraste fase portovenosa axial

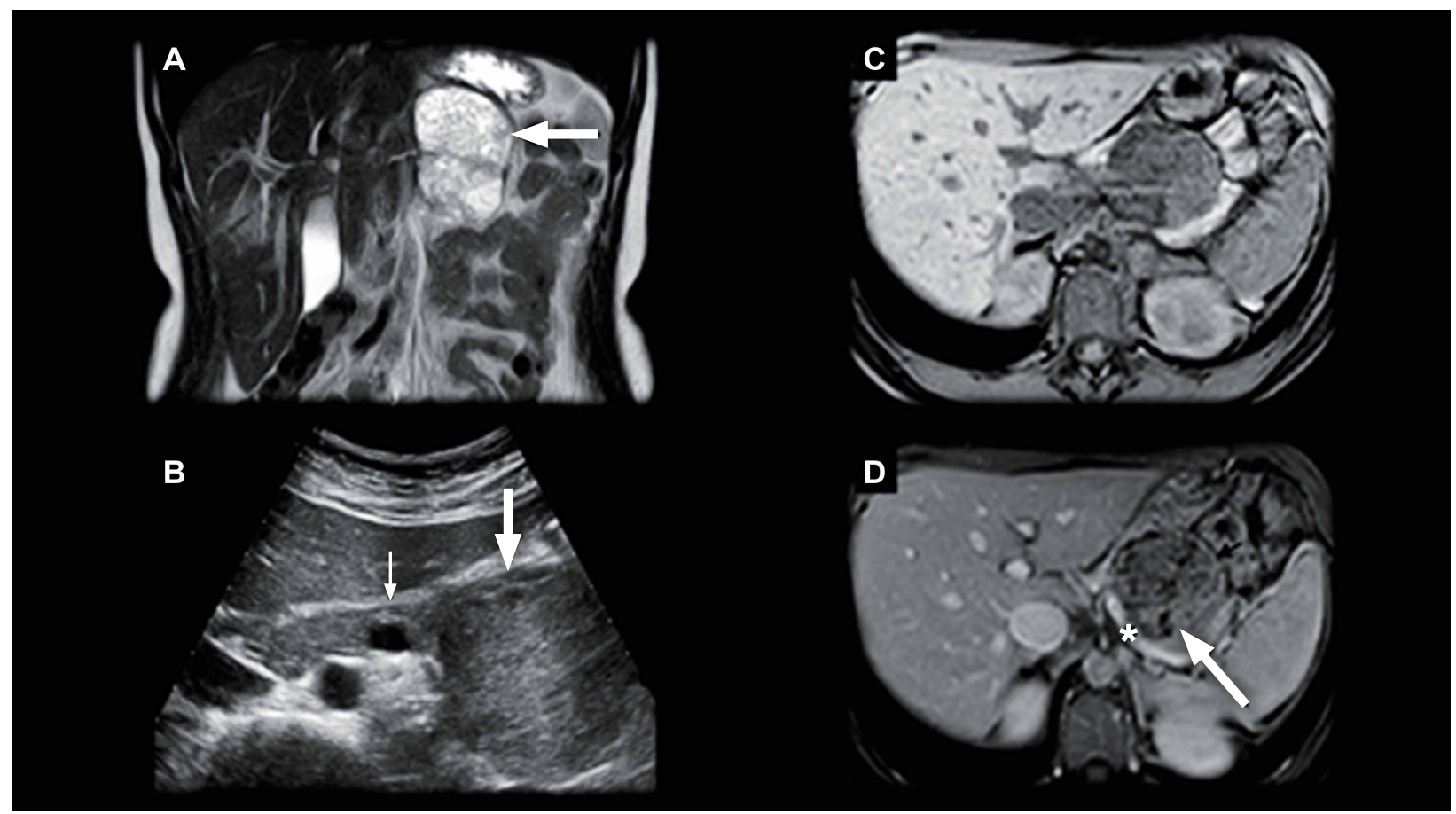

B: Corte axial en el epigastrio a través del páncreas, que demuestra masa heterogénea hipoecogénica bien definida en el cuerpo-cola (flecha grande), que presenta tenue refuerzo acústico posterior que sugiere componentes quísticos en su espesor. Flecha fina: confluencia esplenoporto mesentérica, hito anatómico que define el cuello, delimitando cabeza del cuerpo.

A, C, D: Estudio RM que confirma masa sólido-quística (compleja) variante tipo sólida de SPEN, que reemplaza completamente cuerpo y cola del páncreas, que exhibe alta señal en T2 (A), baja señal en T1 con algunos sutiles focos de ligera alta señal que sugieren material hemático (C), realzando en forma heterogénea por el contraste con fina pseudocápsula periférica (D). Vena esplénica permeable desplazada por la masa, sin signos de infiltración tumoral $\left({ }^{*}\right)$. 
cuyos componentes sólidos restringían en la secuencia de difusión (DWI/ADC), presentando algunos tenues focos de alta señal en secuencias ponderadas en T1 que hacían suponer la presencia de residuos de material hemático en su espesor. Esta masa medía 7,9 x 5,4 x 6,6 cm en sus ejes mayores e indentaba ampliamente la vena esplénica, sin obliterarla ni infiltrarla (Figura 1). El resto del parénquima del páncreas mantenía una arquitectura y señal normales, sin dilatación del conducto pancreático principal, adenopatías retroperitoneales ni ascitis.
Los hallazgos en la RM planteaban en el diagnóstico diferencial, por sus características imagenológicas, entre una NSSP vs. una neoplasia neuroendocrina, por lo que el caso fue presentado al comité institucional hepátobiliar, el cual decidió la resección quirúrgica al no existir signos de diseminación macroscópica a distancia. Se realizó una pancreatectomía distal con esplenectomía vía laparotomía, sin incidentes (Figura 3). La paciente evolucionó favorablemente y fue dada de alta luego de seis días de hospitalización.

Figura 2. Imágenes complementarias de estudio $R M$, que demuestran extensos focos de restricción en difusión $(\boldsymbol{E})$ con correlato en el mapa $A D C(\boldsymbol{F})$ de baja señal que representan alta celularidad (*)

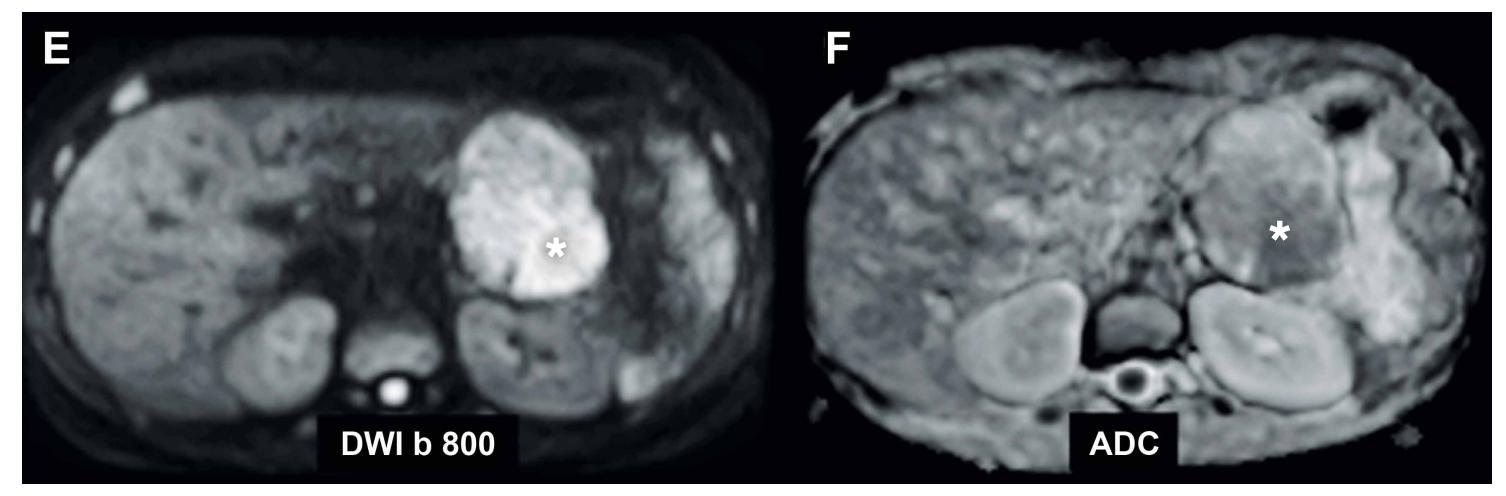

Figura 3. A) Fotografía de pieza quirúrgica: $(\longrightarrow$ ) páncreas normal; $(\longrightarrow$ ) masa en cuerpo-cola del páncreas; $(\overline{\boldsymbol{Y}})$ bazo. B) Fotografia de macroscopia tumoral: $(\Longleftrightarrow)$ focos de hemorragia intratumoral. C) Fotografía de microscopia (HE 40x). Se observan grupos de células disgregadas sin centro conectivo, lo que da un aspecto pseudopapilar, sin atipias

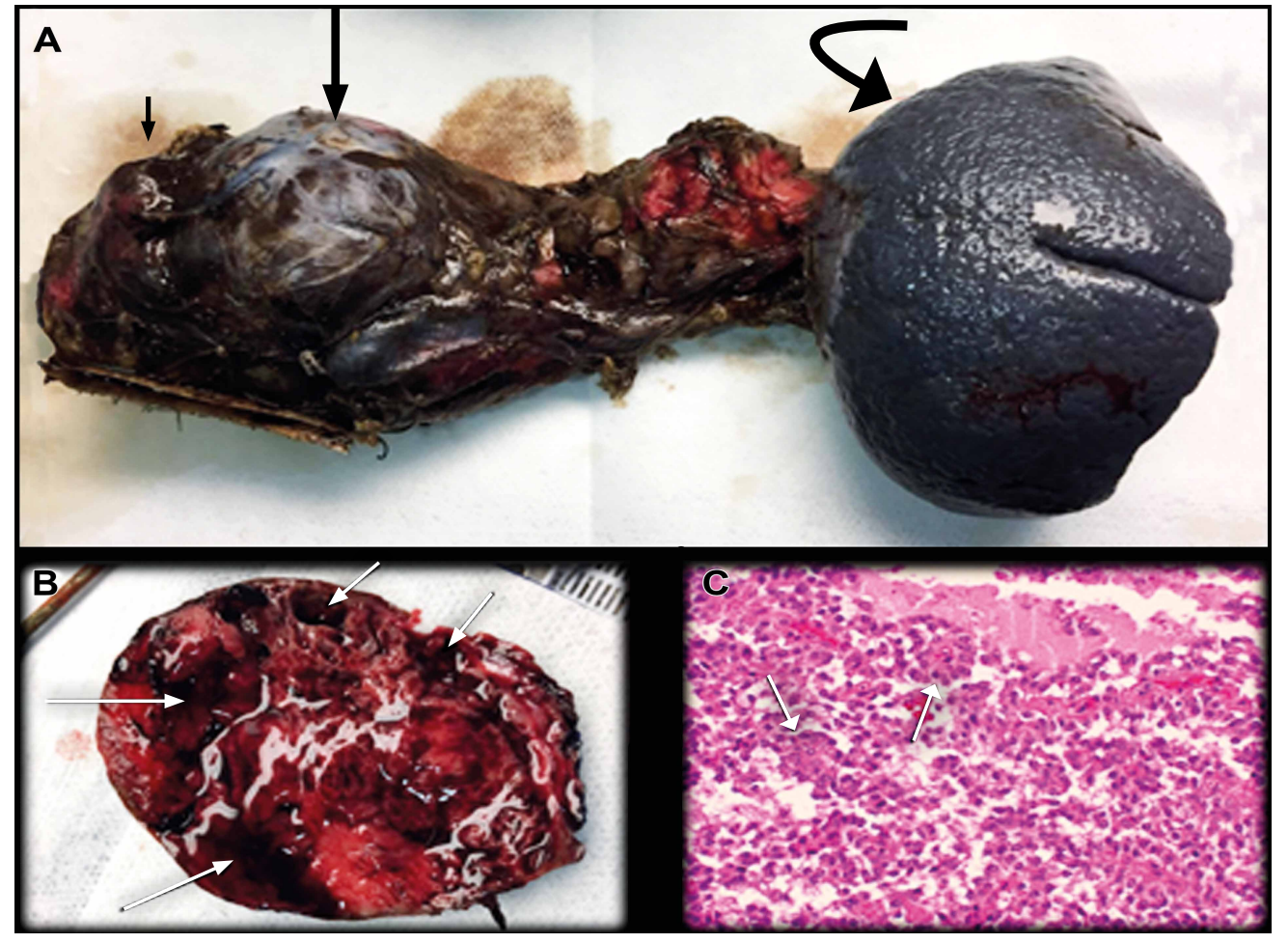


Al estudio histológico, se observó tejido pancreático con desarrollo de un tumor sólido, relativamente bien definido, compuesto por células con escaso citoplasma eosinófilo y núcleos ovoideos claros con ocasionales ranuras, longitudinales pequeños nucléolos y cromatina granular dispersa. Las células se disponen en nidos sólidos alrededor de vasos formando pseudopapilas (Figura 3). Bazo y ganglios linfáticos resecados no demostraron signos de infiltración neoplásica.

\section{Discusión}

Debido a que la mayoría de los pacientes con NSSP presentan síntomas inespecíficos, los estudios imagenológicos cumplen un rol fundamental en el diagnóstico de esta patología de baja incidencia. ${ }^{3,4}$ Además, el aumento global del uso de la tomografía computada multicorte (TCMC) y la RM ha permitido mejorar su diagnóstico en pacientes asintomáticos. ${ }^{3}$

Antiguamente, el estudio imagenológico de elección era la TCMC. ${ }^{1}$ Sin embargo, el desarrollo de la RM y el aumento de su disponibilidad han desplazado la TCMC en la evaluación de los tumores pancreáticos, especialmente en las neoplasias quísticas. ${ }^{1} \mathrm{La}$ RM de abdomen con colangiorresonancia, entregan más información que la TCMC, gracias a la mejor resolución de contraste de los tejidos y a la adecuada caracterización del conducto pancreático principal. ${ }^{1}$

Las NSSP se presentan clásicamente como una voluminosa masa única y bien definida, con áreas de degeneración quística, hemorragia intratumoral y calcificaciones. ${ }^{2,3}$ En RM se observan como una masa quística compleja, con áreas de alta señal en secuencias ponderadas en T2. ${ }^{2}$ Presentan componentes sólidos de disposición en la periferia y cápsula de baja señal en secuencias ponderadas en T1, que realzan en forma gradual y progresiva con el uso de contraste endovenoso. ${ }^{1,2}$ La presencia de áreas de hemorragia intratumoral de alta señal en secuencias ponderadas en T1, constituye uno de los signos más específicos, hallazgo que en el contexto de la edad de presentación (usualmente pacientes adolescentes y adultos jóvenes de sexo femenino) orientan el diagnóstico. ${ }^{2}$ No se asocian a dilatación del conducto pancreático principal ni a atrofia del resto del parénquima pancreático. ${ }^{2}$

De acuerdo al tamaño, las NSSP presentan características imagenológicas particulares, que han sido reportadas en revisiones sistemáticas de esta entidad, distinguiendo entre aquellas menores de $2-3 \mathrm{~cm}$ y mayores de $3 \mathrm{~cm} .{ }^{1,2}$ Los tumores menores de $2-3 \mathrm{~cm}$ suelen ser sólidos, de contornos bien definidos, sin áreas de degeneración quística y/o hemorragia ni pseudocápsula, exhibiendo un escaso realce progresivo con el contraste endovenoso. En
TCMC usualmente son hipodensos en fase no contrastada, pudiendo tornarse isodensos en fase tardía respecto del resto del parénquima pancreático por su impregnación progresiva. En RM demuestran baja señal en T1 y alta señal en T2 por su alto componente de citoplasma. Los tumores mayores de $3 \mathrm{~cm}$ habitualmente se presentan como lesiones sólido-quísticas o de predominio quístico a expensas de las extensas áreas de degeneración quística y/o hemorragia con pseudocápsula periférica continua que tiende a realzar en fases más tardías ${ }^{1,2}$ (Tabla 1).

Tabla 1. Resumen de las principales características SPEN

Conceptos claves

- TU muy baja frecuencia (<3\%).

- Bajo potencial maligno (sobrevida a 5 años 95-100\%).

- MTT hepática - linfática $<20 \%$.

- $90 \%$ sexo fem $<30$ años $(\bar{x}=20$ años).

- Afroamericanos - Asiática > Caucásica.

- Muy buen pronóstico postQx.

- > Afectación cola PNC.

- Gran masa sólida encapsulada con áreas quísticas, sólidas, hemorrágicas.

- Apoyan diagnóstico $\rightarrow$ Cápsula + áreas hemorrágicas intratumoral. No infiltra estructuras vasculares ni dilata Wirsung.

- Tamaño entre 2 - $20 \mathrm{~cm}(\bar{x}=10 \mathrm{~cm})$.

- TU $<2-3 \mathrm{~cm}$ (variante sólida).

En el diagnóstico diferencial, debe considerarse una neoplasia mucinosa que también afecta principalmente a mujeres, presentándose como una lesión de predominio quístico que típicamente se encuentra en la cola del páncreas. ${ }^{4}$ Un tumor neuroendocrino con degeneración quística se diferencia principalmente por su comportamiento hipervascular con el uso de medio de contraste endovenoso. ${ }^{1,2}$ Las metástasis al páncreas, si bien pueden ocurrir en cualquier ubicación dentro de la glándula y con distinta morfología, deben sospecharse cuando el paciente tiene una neoplasia conocida como carcinoma de células renales, melanoma, cáncer de mama, entre otros, y en general, será en pacientes de edad avanzada. El adenocarcinoma es un buen diagnóstico diferencial para las NSPP de pequeño tamaño (variante sólida) con compor- 
tamiento hipovascular con el medio de contraste endovenoso. ${ }^{1,2}$ Aunque el adenocarcinoma es el tumor pancreático más frecuente, este se caracteriza por ser una lesión infiltrativa, excepcionalmente con áreas de degeneración quística (necrosis) o hemorragia, que determina clásicamente dilatación del conducto pancreático principal y/o de la vía biliar común con atrofia parenquimatosa e infiltración vascular. ${ }^{1}$ El pancreatoblastoma, aunque es una entidad casi exclusiva de la población pediátrica, puede presentarse en adolescentes y adultos jóvenes, simulando una NSSP, como una masa de realce heterogéneo, con áreas de degeneración quística. A diferencia del NSSP, suele ubicarse predominantemente en la cabeza, con alteración de las pruebas de laboratorio, arrojando niveles de alfa feto proteína elevada.

El tratamiento de las NSPP consiste en la resección quirúrgica completa, lo que se considera curativo con una tasa de supervivencia de $95 \%$ a cinco años (tasa de mortalidad calculada menor al 2\%). ${ }^{3}$ Menos del $10 \%$ de los pacientes presentan recurrencia o metástasis (generalmente en el hígado), por lo que el seguimiento imagenológico debería continuar al menos por cinco años. ${ }^{3}$
Conflicto de intereses. Los autores niegan conflicto de intereses.

Sostén financiero. Este documento no recibió ningún tipo de financiación o patrocinio externo.

\section{Referencias}

1. Chae SH, Lee JM, Baek JH, et al. Magnetic Resonance Imaging Spectrum of Solid Pseudopapillary Neoplasm of the Pancreas. Journal of Computer Assisted Tomography 2014; 38 (2): 249-257.

2. Ganeshan DM, Paulson E, Tamm EP, Taggart MW, Balachandran A, Bhosale P. Solid pseudo-papillary tumors of the pancreas: current update. Abdominal Imaging 2013; 38 (6): 1373-1382.

3. Law J, Ahmed A, Singh V, Akshintala V, Olson M, Raman S, Ali S, Fishman E, Kamel I, Canto M, Dal Molin M, Moran R, Khashab M, Ahuja N, Goggins M, Hruban R, Wolfgang C, Lennon A. A Systematic Review of Solid-Pseudopapillary Neoplasms. Pancreas 2014; 43 (3): 331-337.

4. Sahani D, Kadavigere R, Saokar A, Fernández del Castillo C, Brugge W, Hahn P. Cystic Pancreatic Lesions: A Simple Imagingbased Classification System for Guiding Management. RadioGraphics 2005; 25 (6): 1471-1484. 